\title{
Robustly stable feedback min-max model predictive control ${ }^{1}$
}

\author{
Eric C. Kerrigan ${ }^{2}$ and Jan M. Maciejowski \\ Department of Engineering, University of Cambridge \\ Trumpington Street, Cambridge CB2 1PZ, United Kingdom \\ Tel: +44-(0)1223-332600, Fax: +44-(0)1223-332662 \\ \{eck21,jmm\}@eng.cam.ac.uk \\ http://www-control.eng.cam.ac.uk
}

\begin{abstract}
This paper is concerned with the practical real-time implementability of robustly stable model predictive control (MPC) when constraints are present on the inputs and the states. We assume that the plant model is known, is discretetime and linear time-invariant, is subject to unknown but bounded state disturbances and that the states of the system are measured. In this paper we introduce a new stage cost and show that the use of this cost allows one to formulate a robustly stable MPC problem that can be solved using a single linear program. Furthermore, this is a multiparametric linear program, which implies that the receding horizon control (RHC) law is piecewise affine, and can be explicitly pre-computed, so that the linear program does not have to be solved on-line.
\end{abstract}

Keywords: min-max problems, robust control, optimal control, receding horizon control, parametric programming, piecewise linear control

\section{Introduction}

In general, solving a feedback min-max problem subject to constraints and disturbances is computationally too demanding for practical implementation. However, various attempts have been made at presenting solutions to this problem. Most of these solutions appear to have come from the field of robust MPC $[13,15]$.

It is by now also well-established that with polytopic disturbance bounds, a linear model and a convex cost, in order to solve finite horizon min-max problems it is sufficient to consider only the disturbance realisations that take on values at the vertices of the disturbance set [16].

As an alternative, in $[3,4,10]$ it is proposed that a dynamicand parametric programming approach be used to obtain an explicit expression for the control law. Provided the stage cost is piecewise affine (e.g. if a 1-norm or $\infty$-norm is used), a piecewise affine expression for the control law can be

\footnotetext{
${ }^{1}$ Research supported by the Royal Academy of Engineering, UK.

${ }^{2}$ Royal Academy of Engineering Post-doctoral Research Fellow.
}

0-7803-7896-2/03/\$17.00 @2003 IEEE computed off-line. However, stability is not proven for the stage and terminal costs proposed in [3,4] nor do the costs satisfy the stability conditions given in $[14, \S 3.3]$ and $[15$, $\S 4.4]$.

The main contribution of this paper is the introduction of a new type of stage cost that can be applied to the results presented in $[3,4,10,16]$ such that robust stability of the closed-loop system is guaranteed. In Section 2 we define in detail the feedback min-max problem that will be considered in this paper and in Section 3 we review known requirements for a receding horizon controller to be robustly stable, and show how the newly-introduced stage cost satisfies these requirements. We also point out some advantages of this cost, over the cost proposed in [10]. In Section 4 we show in detail how the finite-horizon feedback min-max problem can be solved as a single LP, using the results presented in [16], and point out its multi-parametric nature. The conclusions are given in Section 5.

Notation: $\|\cdot\|$ denotes any norm, $d(z, Z):=$ inf $_{y \in Z}\|z-y\|$ for any set $Z \subset \mathbb{R}^{n}, \mathbf{1}:=[1,1, \ldots, 1]^{\prime}$ is a column vector of appropriate length, $:=$ reads "is defined as" and $=$ : reads "defines". For any set $Z, Z^{N}:=\underbrace{Z \times \cdots \times Z}_{N \text { times }}$.

\section{Problem Formulation}

We consider a discrete-time, linear, time-invariant plant

$$
x_{k+1}=A x_{k}+B u_{k}+w_{k}
$$

where $x_{k} \in \mathbb{R}^{n}$ is the system state, $u_{k} \in \mathbb{R}^{m}$ is the control input and $w_{k} \in \mathbf{W}$ is a persistent disturbance that only takes on values in the polytope $\mathbf{W} \subset \mathbb{R}^{n}$. It is assumed that the disturbance $w_{k}$ can jump between arbitrary values within $\mathbf{W}$ and that no stochastic description for it is postulated. Therefore, a worst-case approach is taken in this paper. It is assumed that $(A, B)$ is stabilisable and that polytopic constraints on the state and input, that are either due to physical, safety and/or performance considerations, are also given:

$$
\begin{array}{r}
x_{k} \in \mathbf{X}, \quad u_{k} \in \mathbf{U}, \quad \forall k \in \mathbb{N} . \\
\text { Proceedings of the American Control Conference } \\
\text { Denver, Colorado June 4-6, 2003 }
\end{array}
$$


We assume that $\mathbf{W}$ contains the origin and that $\mathbf{X} \subset \mathbb{R}^{n}$ and $\mathbf{U} \subset \mathbb{R}^{m}$ contain the origin in their interiors.

Since a persistent, unknown disturbance is present, it is impossible to drive the state to the origin. Instead, it is only possible to drive the system to a bounded target set $\mathbf{T}$ contained inside $X$. The goal is to obtain a (time-invariant) nonlinear feedback control law $u=\kappa(x)$ such that the system is robustly steered to the target set, while also satisfying the state and input constraints, and minimising some worst case cost.

In order to determine a suitable control law an optimal control problem $P_{N}$ (defined below) with horizon $N$ is solved. Let $\mathbf{w}:=\left\{w_{0}, w_{1}, \ldots, w_{N-1}\right\}$ denote a disturbance sequence over the interval 0 to $N-1$. Effective control in the presence of the disturbance requires state feedback [15, §4.6], so that the decision variable in the optimal control problem (for a given initial state) is a control policy $\pi$ defined by

$$
\pi:=\left\{u(0), \mu_{1}(\cdot), \ldots, \mu_{N-1}(\cdot)\right\},
$$

where $u(0) \in \mathbf{U}$ and $\mu_{k}: \mathbf{X} \rightarrow \mathbf{U}, k=1, \ldots, N-1 ; u(0)$ is a control action (since the current state is known) and each $\mu_{k}(\cdot)$ is a state feedback control law. Let $\phi(k ; x, \pi, \mathbf{w})$ denote the solution to (1) at time $k$ when the state is $x$ at time 0 , the control is determined by policy $\pi\left(u=\mu_{k}(x)\right.$ at event $(x, k)$, i.e. state $x$, time $k$ ) and the disturbance sequence is $\mathbf{w}$.

Given a target set (often also called terminal constraint set) $\mathbf{T} \subset \mathbf{X}$ containing the origin, for each initial state $x \in \mathbf{X}$, let $\Pi_{N}(x)$ denote the set of admissible policies, i.e.

$$
\begin{aligned}
\Pi_{N}(x):= & \left\{\pi \mid u(0) \in \mathbf{U}, \mu_{k}(\phi(k ; x, \pi, \mathbf{w})) \in \mathbf{U},\right. \\
\phi(k ; x, \pi, \mathbf{w}) & \in \mathbf{X}, \phi(N ; x, \pi, \mathbf{w}) \in \mathbf{T}, \\
& \left.\forall k \in\{1, \ldots, N-1\}, \forall \mathbf{w} \in \mathbf{W}^{N}\right\}
\end{aligned}
$$

and let

$$
X_{N}:=\left\{x \in \mathbf{X} \mid \Pi_{N}(x) \neq \emptyset\right\}
$$

denote the set of states in $\mathbf{X}$ that can be robustly steered (steered for all $\mathbf{w} \in \mathbf{W}^{N}$ ) to the target set $\mathbf{T}$ in $N$ steps.

In order to define an optimal control problem, a cost $V_{N}(\cdot)$ that is dependent on the policy $\pi$ and current state $x$, but not dependent on $\mathbf{w}$, is defined; the conventional choice is

$$
V_{N}(x, \pi):=\max _{\mathbf{w} \in W^{N}}\left[\sum_{k=0}^{N-1} L\left(x_{k}, u_{k}\right)+F\left(x_{N}\right)\right]
$$

where $x_{k}:=\phi(k ; x, \pi, \mathbf{w}) \quad$ if $k \in\{0, \ldots, N\}, \quad u_{k}:=$ $\mu_{k}(\phi(i ; x, \pi, \mathbf{w}))$ if $k \in\{1, \ldots, N-1\}$ and $u_{0}:=u(0)$.

The target set $\mathbf{T}$, stage cost $L(\cdot)$ and terminal cost $F(\cdot)$ have to satisfy certain conditions in order to ensure that the solution of the feedback min-max optimal control problem, when implemented in a receding horizon fashion, is robustly stabilising. These conditions will be set out in the following section.

The feedback min-max optimal control problem $P_{N}$ can now be defined as

$$
P_{N}(x): \quad V_{N}^{*}(x):=\min _{\pi}\left\{V_{N}(x, \pi) \mid \pi \in \Pi_{N}(x)\right\} .
$$

Let $\pi_{N}^{*}(x)=:\left\{u_{0}^{*}(x), \mu_{1}^{*}(\cdot ; x), \ldots, \mu_{N-1}^{*}(\cdot ; x)\right\}$ denote the solution to $P_{N}(x)$, i.e.

$$
\pi_{N}^{*}(x):=\arg \min _{\pi}\left\{V_{N}(x, \pi) \mid \pi \in \Pi_{N}(x)\right\},
$$

where the notation $\mu_{i}^{*}(\cdot ; x)$ shows the dependence of the optimal policy on the current state $x$.

It should be noted that the solution to problem $P_{N}$ is frequently not unique - that is, there can be a whole set of minimisers, from which one must be selected. Thus the time-invariant, set-valued receding horizon control (RHC) law $\kappa_{N}: X_{N} \rightarrow 2^{U}\left(2^{U}\right.$ is the set of all subsets of $U$ ) is defined by the first element of $\pi_{N}^{*}(x)$ :

$$
\kappa_{N}(x):=u_{0}^{*}(x), \quad \forall x \in X_{N} .
$$

Typically, but not always, $u_{0}^{*}(x)$ is a singleton.

The feedback min-max problem $P_{N}$ defined in (6) is an infinite dimensional optimisation problem and impossible to solve directly. However, methods for solving $P_{N}$ using finite dimensional optimisation techniques have been proposed in $[3,4,10,16]$ and this paper can be seen as an immediate extension of [16].

Before proceeding, some comments regarding the choice of stage cost are in order. Robust stability can be guaranteed if the stage cost

$$
L(x, u):=\left\{\begin{array}{ll}
\|Q x\|+\|R u\| & \text { if }(x, u) \in(\mathbf{X} \backslash \mathbf{T}) \times \mathbf{U} \\
0 & \text { if }(x, u) \in \mathbf{T} \times \mathbf{U}
\end{array},\right.
$$

proposed in [10,14], is used. Though this choice of cost solves the stability problem, it should be noted that (9) is not continuous (on the boundary of $\mathbf{T}$ ). The use of such a discontinuous stage cost is a major obstacle to implementation using standard solvers for linear, quadratic, semi-definite or other smooth, convex nonlinear programming problems. As such, a new cost (defined below) is proposed as an alternative that solves the problem of obtaining a continuous stage cost that can be implemented using smooth, convex programming solvers, while still guaranteeing robust stability of the closed-loop system.

In this paper, we introduce a new type of stage cost:

$$
L(x, u):=\min _{y \in \mathbf{T}}\|Q(x-y)\|_{p}+\|R(u-K x)\|_{p},
$$

where $Q \in \mathbb{R}^{n \times n}$ and $R \in \mathbb{R}^{m \times m}$ are weights, $K \in \mathbb{R}^{m \times n}$ is a linear feedback gain and $\mathbf{T} \subset \mathbb{R}^{n}$ is a polytope containing the origin. We will show that, if $p=1$ or $p=\infty$, the use of this stage cost allows the robustly stable feedback min-max MPC problem to be solved using a single linear program (LP). Furthermore, we will show that this LP is in fact a multi-parametric LP (mp-LP), that allows the RHC law $\kappa_{N}(\cdot)$ to be pre-computed off-line along the lines developed in $[2,5]$, and from which it follows that this law is in fact piecewise affine. These facts make robust MPC/RHC, using the stage cost (10), a viable proposition for some realistic problems. 
Remark 1 A similar stage cost to (10) was independently proposed in [12] and briefly discussed within the context of guaranteeing robust stability of a new type of MPC scheme. The stage cost proposed in [12] is $L(x, u):=(1 / 2) \| x-$ $\operatorname{Proj}_{\mathbf{T}}(x)\left\|_{2}^{2}+(1 / 2)\right\| u-K x \|_{2}^{2}$, where $\operatorname{Proj}_{\mathbf{T}}(x)$ denotes the orthogonal projection of $x$ onto $\mathrm{T}$. The difference between this stage cost and (10) is minor, but the formulation in (10) is perhaps more natural.

Remark 2 This paper investigates the use of (10) in solving $P_{N}$ using the method proposed in [16]. Though not discussed here, it is possible to use (10) in solving $P_{N}$ using the methods described in $[3,4,10]$.

\section{Requirements for Robust Stability}

It is well-known that, for an MPC/RHC law that assumes a finite horizon, an arbitrary choice of terminal constraint, stage cost and terminal cost does not guarantee stability of the closed-loop system. In the absence of state disturbances, conventional MPC/RHC schemes employ a terminal cost $F(x):=\|P x\|$, that is a control Lyapunov function inside $\mathbf{T}$, in order to guarantee robust stability of the origin for the closed-loop system $[14,15]$. However, if the interior of $\mathbf{W}$ is non-empty and the disturbance is persistent, then one can easily show that there does not exist a so-called robust control Lyapunov function in a neighbourhood of the origin. Since it is no longer possible to drive the system to the origin, but only to some set containing the origin, the conventional choice of stage and terminal cost cannot guarantee stability or convergence $[14, \S 3.3 .2]$ and a new type of stage and terminal cost is needed.

The following definitions are taken from [10]: The set $\mathbf{T}$ is robustly stable iff, for all $\varepsilon>0$, there exists a $\delta>0$ such that $d\left(x_{0}, \mathbf{T}\right) \leq \delta$ implies $d\left(x_{i}, \mathbf{T}\right) \leq \varepsilon$, for all $i \geq 0$ and all admissible disturbance sequences. The set $\mathbf{T}$ is robustly asymptotically (finite-time) attractive with domain of attraction $X$ iff for all $x_{0} \in X, d\left(x_{i}, \mathbf{T}\right) \rightarrow 0$ as $i \rightarrow \infty$ (there exists a time $M$ such that $x_{i} \in \mathbf{T}$ for all $i \geq M$ ) for all admissible disturbance sequences. The set $\mathbf{T}$ is robustly asymptotically (finite-time) stable with domain of attraction $X$ iff it is robustly stable and robustly asymptotically (finite-time) attractive with domain of attraction $X$.

Consider now the following assumptions, adapted from [10, 16, 17]:

A1: The terminal constraint set $\mathbf{T} \subset \mathbf{X}$ contains the origin in its interior. A linear, time-invariant control law $K: \mathbb{R}^{n} \rightarrow \mathbb{R}^{m}$ is given such that the terminal constraint set $\mathbf{T}$ is disturbance invariant [11] under the control $u=K x$, i.e. $(A+B K) x+w \in \mathbf{T}$ for all $x \in \mathbf{T}$ and all $w \in \mathbf{W}$. In addition, $K x \in \mathrm{U}$ for all $x \in \mathrm{T}$.

A2: The terminal cost $F(x):=0$ for all $x \in \mathbb{R}^{n}$.

A3: The stage cost $L(x, u):=0$ if $x \in \mathbf{T}$ and $u=K x$.

A4a: $L(\cdot)$ is continuous over $\mathbf{X} \times \mathbf{U}$ and there exists a $c>0$ such that $L(x, u) \geq c(d(x, \mathbf{T}))$ for all $(x, u) \in(\mathbf{X} \backslash \mathbf{T}) \times \mathbf{U}$.
A4b: $L(\cdot)$ is continuous over $(\mathbf{X} \backslash \mathbf{T}) \times \mathbf{U}$ and there exists a $c>0$ such that $L(x, u) \geq c\|x\|$ for all $(x, u) \in(\mathbf{X} \backslash \mathbf{T}) \times \mathbf{U}$.

$\mathrm{A} 1, \mathrm{~A} 2, \mathrm{~A} 3, \mathrm{~A} 4 \mathrm{a}$ and $\mathrm{A} 4 \mathrm{~b}$ satisfy the assumptions on the stage cost, terminal cost and terminal constraint given in $[14, \S 3.3]$ and $[15, \S 4.4]$. Hence, one can follow a standard procedure of using the optimal value function as a candidate Lyapunov function $[14,15]$ and show that:

Theorem 1 If A1, A2, $A 3$ and $A 4 a$ (and $A 4 b$ ) hold, then $\mathbf{T}$ is robustly asymptotically (finite-time) stable for the closedloop system $x_{k+1}=A x_{k}+B \kappa_{N}\left(x_{k}\right)+w_{k}$ with a region of attraction $X_{N}$.

Consider also the "dual-mode" control law

$$
\Gamma(x):= \begin{cases}\kappa_{N}(x) & \text { if } x \in X_{N} \backslash \mathbf{T} \\ K x & \text { if } x \in \mathbf{T}\end{cases}
$$

where $\kappa_{N}(\cdot)$ is defined in (8). If $\mathbf{T}, K, F(\cdot)$ and $L(\cdot)$ are chosen such that assumptions A1, A2, A3 and A4 are satisfied, then $\Gamma(\cdot)$ is clearly also a robustly stabilising control law, by Theorem 1.

In $[15, \S 4.6 .3]$ and [16] it is argued that one need only consider the set of extreme disturbance realisations if the following assumption holds in addition to those given above:

\section{A5: $L(\cdot)$ is convex over $\mathbf{X} \times \mathbf{U}$.}

It is shown in [16] how, provided A1, A2, A3, A4a (and $\mathrm{A} 4 \mathrm{~b}$ ) and $\mathrm{A} 5$ hold, one can associate a different control input sequence with each extreme disturbance realisation and, using a causality constraint that prevents the optimiser from assuming knowledge of future disturbances, one can compute a control input $u \in \kappa_{N}(x)$ on-line using standard finitedimensional convex programming solvers. However, in [15, \$4.6.3] and [16], an exact expression for the stage cost that allows one to implement the proposed method is not given; only general conditions on $L(\cdot)$ as in $\mathrm{A} 3, \mathrm{~A} 4 \mathrm{a}$ and $\mathrm{A} 4 \mathrm{~b}$ are given.

Our main concern here is to point out that if $Q$ is nonsingular, then the stage cost (10) satisfies assumptions A3 and $\mathrm{A} 4 \mathrm{a}$ (but not $\mathrm{A} 4 \mathrm{~b}$ ). Using this stage cost in computing $\kappa_{N}(\cdot)$ thus assures that $\mathbf{T}$ is robustly asymptotically stable (but not necessarily finite-time stable) for the closed-loop system. Additional assumptions, which guarantee that $\mathbf{T}$ is robustly finite-time stable, can be found in [9].

Furthermore, the stage cost (10) satisfies assumption A5 if $\mathbf{T}$ is convex (for proof, see [9]). Its use thus allows problem $P_{N}$ to be solved as a finite-dimensional problem, as will be shown in more detail in the next section.

Remark 3 We once again point out that the stage cost (9), that was proposed in $[10,14]$, is not continuous and hence not convex. As such, it does not satisfy assumption A5 and therefore cannot be used with the approach proposed in [16].

The choice of $K$ in (10) is problem-dependent, but typically it is chosen such that $A+B K$ has all its eigenvalues strictly 
inside the unit disk and the control $u=K x$ is optimal with respect to some performance measure. The exact choice of $\mathbf{T}$ is also problem-dependent, but a sensible choice for $\mathbf{T}$ is the minimal or maximal disturbance invariant set [11]. For methods of computing a $\mathbf{T}$ that satisfies $\mathrm{A} 1$, see $[11,16]$, and for a further discussion regarding the choice of $\mathbf{T}$, see [9].

Finally, it is worth pointing out that, provided $Q$ is nonsingular, A3 and A4a are satisfied even if $R$ is singular or $R:=0$ in (10). As such, the use of the second term is not necessary in guaranteeing robust stability and only affects the performance of the closed-loop system.

\section{Solution via Linear Programming}

Following the same approach as the one taken in [16], let $\mathbf{w}^{\ell}:=\left\{w_{0}^{\ell}, \ldots, w_{N-1}^{\ell}\right\}$ denote an allowable disturbance sequence over the finite horizon $k=0, \ldots, N-1$ and let $\ell \in L$ index these realisations (this is a slight abuse of notation, because the set of possible realisations is uncountable). Also let $\mathbf{u}^{\ell}:=\left\{u_{0}^{\ell}, \ldots, u_{N-1}^{\ell}\right\}$ denote a control sequence associated with the $\ell$ 'th disturbance realisation and let $\mathbf{x}^{\ell}:=\left\{x_{0}^{\ell}, \ldots, x_{N}^{\ell}\right\}$ represent the sequence of solutions of the model equation

$$
x_{k+1}^{\ell}=A x_{k}^{\ell}+B u_{k}^{\ell}+w_{k}^{\ell}, \ell \in L
$$

with $x_{0}^{\ell}=x$, where $x$ denotes the current state.

Let the finite subset $L_{v} \subset L$ index those disturbance sequences $\mathbf{w}^{\ell}$ that take on values at the vertices of the polytope $\mathbf{W}^{N}$. Also, let the sẹt of input sequences associated with the set of extreme disturbance realisations be

$$
\mathbf{u}:=\left\{\mathbf{u}^{1}, \mathbf{u}^{2}, \ldots, \mathbf{u}^{v}\right\},
$$

where $V$ is the cardinality of $L_{v}$.

As a first step towards an implementable solution we follow [16] in replacing problem $P_{N}$ by the following finitedimensional problem, in which the optimisation is over control sequences associated with extreme disturbance realisations, but with a so-called causality constraint:

\section{Problem 1 (Finite-dimensional feedback min-max)}

Given the current state $x$, find a solution to the problem

$$
\mathbf{u}^{*}(x):=\left(\arg \min _{\mathbf{u}}\right) \max _{\ell \in L_{v}}\left[F\left(x_{N}^{\ell}\right)+\sum_{k=0}^{N-1} L\left(x_{k}^{\ell}, u_{k}^{\ell}\right)\right]
$$

such that for all $\ell \in L_{v}$ and $k \in\{0, \ldots, N-1\}$,

$$
\begin{gathered}
x_{k+1}^{\ell}=A x_{k}^{\ell}+B u_{k}^{\ell}+w_{k}^{\ell}, \quad x_{0}^{\ell}=x, \\
u_{k}^{\ell} \in \mathbf{U}, \quad x_{k}^{\ell} \in \mathbf{X}, \quad x_{N}^{\ell} \in \mathbf{T}, \\
x_{k}^{\ell_{1}}=x_{k}^{\ell_{2}} \Rightarrow u_{k}^{\ell_{1}}=u_{k}^{\ell_{2}}, \quad \forall \ell_{1}, \ell_{2} \in L_{v} .
\end{gathered}
$$

Note the following: (i) a different control input sequence is associated with each disturbance sequence, thereby overcoming the problem of open-loop MPC [1, 7] that associates a single control input sequence with all disturbance sequences; (ii) the causality constraint (13d) associates with each $x_{k}^{\ell}$ a single control input, thereby reducing the degrees of freedom and making the control law independent of the control and disturbance sequence taken to reach that state.

If one lets

$$
\mathbf{u}^{*}(x)=:\left\{\mathbf{u}^{1 *} \cdot(x), \mathbf{u}^{2 *}(x), \ldots, \mathbf{u}^{V *}(x)\right\},
$$

then the question one can now ask is under what conditions the first component of $\mathbf{u}^{1 *}(x)$, denoted by $u_{0}^{1 *}(x)$, is equal to $\kappa_{N}(x)$ (recall that (13d) ensures that the first components of all the $\mathbf{u}^{\ell *}(x), \ell \in L_{v}$, are equal). As noted in [15, $\left.\S 4.6 .3\right]$, if the system is linear, $\mathbf{X}, \mathbf{U}, \mathbf{W}$ and $\mathbf{T}$ are polytopes and $F(\cdot)$ and $L(\cdot)$ are convex functions, then using similar convexity arguments as in [16, Thm. 2], it can be shown that the first element of $\mathbf{u}^{1 *}(x)$ is equal to $\kappa_{N}(x)$. The next result follows:

\section{Theorem 2 (Robustly stable feedback min-max RHC)} Suppose AI, A2 and A3 are satisfied. If the stage cost is given by (10) and $Q$ is non-singular, then $\kappa_{N}(x)=u_{0}^{1 *}(x)$ and $\mathbf{T}$ is robustly asymptotically stable for the closedloop system $x_{k+1}=A x_{k}+B \kappa_{N}\left(x_{k}\right)+w_{k}$ with a region of attraction $X_{N}$.

At first sight, it might not be clear how the the causality constraint (13d) translates into linear constraints. However, note that for all $k \in\{0, \ldots, N-2\}$ and $\ell_{1}, \ell_{2} \in L_{v}$, if $x_{0}^{\ell_{1}}=x_{0}^{\ell_{2}}, w_{j}^{\ell_{1}}=w_{j}^{\ell_{2}}$ and $u_{j}^{\ell_{1}}=u_{j}^{\ell_{2}}$ for all $j \in\{0, \ldots, k\}$, then $x_{j}^{\ell_{1}}=x_{j}^{\ell_{2}}$ for all $j \in\{1, \ldots, k+1\}$. Hence one needs to set $u_{k+1}^{\ell_{1}}=u_{k+1}^{\ell_{2}}$ in order to satisfy the causality constraint. Therefore, as discussed in $[13,16]$, the causality constraint (13d) can be replaced by associating the same control input with each node of the resulting extreme disturbance/state trajectory tree. This observation reduces the original number of control inputs that need to be computed from $N v^{N}$ to $1+v+\ldots+v^{N-1}$, where $v$ is the number of vertices of $\mathbf{W}$. A similar observation holds for the number of constraints and slack variables that need to be considered.

As a small example, consider the case when $v=2$ and $N=$ 2. There are $V=v^{N}=4$ extreme disturbance sequences and if $L_{v}$ has been defined such that $w_{0}^{1}=w_{0}^{2}$ and $w_{0}^{3}=w_{0}^{4}$, then (13d) can be substituted with $u_{0}^{1}=u_{0}^{2}=u_{0}^{3}=u_{0}^{4}, u_{1}^{1}=$ $u_{1}^{2}$ and $u_{1}^{3}=u_{1}^{4}$.

Clearly, the number of decision variables and constraints grows exponentially with the length of the control horizon. Implementing robust MPC formulated along these lines with large control horizons is therefore questionable. However, for some problems the computational complexity might still be acceptable.

\subsection{Setting up as an LP problem}

In [16] it was suggested that the solution to (13) should be computed on-line using standard convex, nonlinear programming solvers. We will now describe how this problem can be solved using linear programming if stage cost (10) is used. This will involve setting up a linear program that is equivalent to (13). 
Recalling that $F(x):=0$, let the total cost $J\left(x, \mathbf{u}^{\ell}, \mathbf{w}^{\ell}\right)$ for the current state $x$ and a sequence of control inputs $\mathbf{u}^{\ell}$ associated with a given disturbance realisation $\mathbf{w}^{\ell}$ be defined as

$$
J\left(x, \mathbf{u}^{\ell}, \mathbf{w}^{\ell}\right):=\sum_{k=0}^{N-1} L\left(x_{k}^{\ell}, u_{k}^{\ell}\right)
$$

As in [16], the optimisation problem in (13) can be written as

$$
\min _{\mathbf{u} \in \mathbf{C}(x)} \max _{\ell \in L_{v}} J\left(x, \mathbf{u}^{\ell}, \mathbf{w}^{\ell}\right),
$$

where $\mathbf{C}(x)$ is a polytope implicitly defined by the constraints in (13). Clearly, the optimisation in (14) is equivalent to the convex program

$$
\min _{\mathbf{u}, \gamma}\left\{\dot{\gamma} \mid \mathbf{u} \in \mathbf{C}(x), J\left(x, \mathbf{u}^{\ell}, \mathbf{w}^{\ell}\right) \leq \gamma, \forall \ell \in L_{v}\right\} .
$$

Before proceeding, note that if one uses the stage cost (10) with $p=1$ then the value of $\min _{u \in \mathbf{U}} L(x, u)$ can be computed by solving the linear program

$$
\min _{u \in \mathbf{U}} L(x, u)=\min _{u, y, \alpha, \beta} \mathbf{1}^{\prime} \alpha+\mathbf{1}^{\prime} \beta
$$

subject to

$$
\begin{aligned}
-\alpha \leq Q(x-y) \leq \alpha, & y \in \mathbf{T}, \\
-\beta \leq R(u-K x) \leq \beta, & u \in \mathbf{U},
\end{aligned}
$$

where the vectors $\alpha \in \mathbb{R}^{n}$ and $\beta \in \mathbb{R}^{m}$.

The above procedure is fairly standard and has been used in converting standard and open-loop min-max MPC problems with 1 -norm and $\infty$-norm costs to linear programs $[1,2,7$, 13]. We now use it to set up a linear program equivalent to (13). Let

$$
J\left(x, \mathbf{u}^{\ell}, \mathbf{w}^{\ell}\right):=\min _{\mathbf{y}^{\ell}} \sum_{k=0}^{N-1}\left\|Q\left(x_{k}^{\ell}-y_{k}^{\ell}\right)\right\|_{1}+\left\|R\left(u_{k}^{\ell}-K x_{k}^{\ell}\right)\right\|_{1},
$$

and $\mathbf{y}^{\ell}, \mu^{\ell}, \eta^{\ell}$ and $\mathbf{y}, \mu, \eta$ be defined similarly to $\mathbf{u}^{\ell}$ and $\mathbf{u}$. It now follows that $(13)$ is equivalent to

$$
\min _{\mathbf{u}, \mathbf{y}, \mu, \eta, \gamma} \gamma
$$

such that for all $\ell \in L_{v}$ and $k \in\{0, \ldots, N-1\}$,

$$
\begin{array}{r}
x_{k+1}^{\ell}=A x_{k}^{\ell}+B u_{k}^{\ell}+w_{k}^{\ell}, \quad x_{0}^{\ell}=x, \\
-\mu_{k}^{\ell} \leq Q\left(x_{k}^{\ell}-y_{k}^{\ell}\right) \leq \mu_{k}^{\ell}, \quad y_{k}^{\ell} \in \mathbf{T}, \\
-\eta_{k}^{\ell} \leq R\left(u_{k}^{\ell}-K x_{k}^{\ell}\right) \leq \eta_{k}^{\ell}, \quad u_{k}^{\ell} \in \mathbf{U}, \\
x_{k}^{\ell} \in \mathbf{X}, \quad x_{N}^{\ell} \in \mathbf{T}, \\
\sum_{k=0}^{N-1} 1^{\prime} \mu_{k}^{\ell}+1^{\prime} \eta_{k}^{\ell} \leq \gamma, \\
x_{k}^{\ell_{1}}=x_{k}^{\ell_{2}} \Rightarrow u_{k}^{\ell_{1}}=u_{k}^{\ell_{2}}, \quad \forall \ell_{1}, \ell_{2} \in L_{v} .
\end{array}
$$

Note that it is also possible to convert (13) to a linear program if $p=\infty$ is chosen in the stage cost (10). This is achieved in a similar fashion as above by noting that if $L(x, u):=\min _{y \in \mathbf{T}}\|Q(x-y)\|_{\infty}+\|R(u-K x)\|_{\infty}$, then

$$
\min _{u \in \mathbf{U}} L(x, u)=\min _{u, y, \alpha, \beta} \alpha+\beta
$$

subject to

$$
\begin{aligned}
-1 \alpha \leq Q(x-y) \leq 1 \alpha, & y \in \mathrm{T}, \\
-1 \beta \leq R(u-K x) \leq 1 \beta, & u \in \mathrm{U},
\end{aligned}
$$

where the scalars $\alpha \in \mathbb{R}$ and $\beta \in \mathbb{R}$.

It is interesting to observe that the use of the $\infty$-norm results in less variables and constraints than in the case of the 1-norm. The former choice of norm is therefore probably preferred if computational speed is an issue. However, the latter norm might be preferred if a control action is sought that is closer to having used the quadratic norm, as in conventional MPC.

\subsection{Explicit solution of the RHC law via parametric pro- gramming}

The development in the previous section allows the on-line solution of the robust MPC problem, providing that the available computing resources and the required update interval are such that the LP can be solved quickly enough. If this is not possible, an alternative is to pre-compute the solution, to store this solution in a database, and to read out the appropriate part of the solution (which can be done relatively quickly) as required.

By substituting (16b) into the rest of the constraints it is possible to show, as in $[2,5]$, that $(16)$ can be written in the form

$$
\min _{\theta}\left\{c^{\prime} \theta \mid F \theta \leq g+G x\right\},
$$

where $\theta$ is the decision variable that consists of the nonredundant components of $(\mathbf{u}, \mathbf{y}, \mu, \eta, \gamma)$; the vectors $c, g$ and matrices $F, G$ are of appropriate dimensions and do not depend on $x$. The key observation here is that the constraints are dependent on the current state $x$ in the affine manner shown above. This means that the feedback min-max problem falls into the class of multi-parametric linear programs (mp-LPs) $[6,8]$, where each component of $x$ represents a parameter that will affect the solution. This class of problems can be solved off-line for all allowable values of $x$ and results in a piecewise affine expression for the solution in terms of $x[6,8]$.

The polyhedron $X_{N}=\left\{x \in \mathbb{R}^{n} \mid \exists \theta: F \theta \leq g+G x\right\}$ is the set of states for which a solution to (17) exists. Given a polytope of states $\mathfrak{X} \subseteq X_{N}$ and using the algorithm described in [6], one can compute the explicit expression of the feedback min-max RHC law for all $x \in \mathfrak{X}$. The resulting feedback min-max RHC law is then of the following piecewise affine form:

$$
\kappa_{N}(x)=K_{i} x+h_{i}, \text { if } x \in \mathfrak{X}_{i},
$$

where each matrix $K_{i} \in \mathbb{R}^{m \times n}$ and vector $h_{i} \in \mathbb{R}^{m}$ are associated with a polytope $\mathfrak{X}_{i}$. The set of polytopes $\left\{\mathfrak{X}_{i}\right\}$ have 
mutually disjoint interiors and $\mathfrak{X}=\bigcup_{i} \mathfrak{X}_{i}$. All that is required on-line is to determine in which critical region the current state lies and then compute the control action using only matrix multiplication and addition.

The solution to the control law presented here is of the same piecewise affine structure as the one given in $[3,4]$. However, the derivation in [3] requires the solution of $2 \mathrm{~N}$ multiparametric mixed-integer linear programs (mp-MILPs). By exploiting the convex, piecewise affine nature of the optimal cost, this has since been improved to solving $N$ mp-LPs [4]. The result presented in this paper requires the solution of a single mp-LP instead, though this is perhaps of more significance for the on-line computation than for off-line precomputation of the RHC law.

Finally, we once again mention that robust stability is not guaranteed for the stage cost used in [3, 4]. However, robust stability in $[3,4]$ can be guaranteed using the new stage cost $(10)$ proposed in this paper.

\section{Conclusions}

Robust MPC requires optimisation over feedback policies, rather than the more traditional optimisation over open-loop sequences, if excessive conservativeness, and hence infeasibility and/or instability, is to be avoided. But this is difficult to implement with reasonable computational effort, and hence its practicality has been questionable, particularly if on-line optimisation in real-time is envisaged.

In this paper we have introduced a new stage cost, which allows one to compute the solution of the full robust receding horizon control problem - that is, optimisation over feedback policies with guaranteed robust convergence to the target set in the face of persistent disturbances - using only one linear program. This is in contrast with previous proposals that have required the solution of nonlinear programs and/or the solution of a number of optimisation problems.

A detailed comparison of the competing proposals is not straightforward, however, because the dimensions of the optimisations involved vary in complicated ways. It is therefore not yet possible to say conclusively which scheme will be more efficient for on-line implementation, or which one would be preferred for off-line pre-computation. The answers may well depend on problem-specific details.

\section{References}

[1] J.C. Allwright and G.C. Papavasiliou. On linear programming and robust model-predictive control using impulse-responses. Systems \& Control Letters 18 (1992) 159-164.

[2] A. Bemporad, F. Borrelli and M. Morari. Model predictive control based on linear programming - The ex- plicit solution. IEEE Trans. Automatic Control 47:12 (2002) 1974-1985.

[3] A. Bemporad, F. Borrelli and M. Morari. Robust model predictive control: Piecewise linear explicit solution. In Proc. European Control Conference, Porto, Portugal (2001).

[4] A. Bemporad, F. Borrelli and M. Morari. Min-max control of constrained uncertain discrete-time linear systems. IEEE Trans. Automatic Control (in press).

[5] A. Bemporad, M. Morari, V. Dua and E.N. Pistikopoulos. The explicit linear quadratic regulator for constrained systems. Automatica 38:1 (2002) 3-20.

[6] F. Borrelli, A. Bemporad and M. Morari. A geometric algorithm for multi-parametric linear programming. Journal of Optimization Theory and Applications (in press).

[7] P.J. Campo and M. Morari. Robust model predictive control. In Proc. American Control Conference, Green Valley AZ, USA (1987) 1021-1026.

[8] T. Gal. Postoptimal Analyses, Parametric Programming, and Related Topics. 2nd Edition, de Gruyter, 1995.

[9] E.C. Kerrigan and J.M. Maciejowski. Feedback minmax model predictive control using a single linear program: Robust stability and the explicit solution. International Journal of Robust and Nonlinear Control (in press).

[10] E.C. Kerrigan and D.Q. Mayne. Optimal control of constrained, piecewise affine systems with bounded disturbances. In Proc. 41st IEEE Conference on Decision and Control, Las Vegas NV, USA (2002).

[11] I. Kolmanovsky and E.G. Gilbert. Theory and computation of disturbance invariant sets for discrete-time linear systems. Mathematical Problems in Engineering: Theory, Methods and Applications 4 (1998) 317-367.

[12] W. Langson, I. Chryssochoos and D. Mayne. Robust model predictive control using tubes. Technical Report EEE/C\&P/DQM/11/2001, Imperial College, London, UK (2001). Submitted to Automatica.

[13] J.M. Maciejowski. Predictive Control with Constraints. Prentice Hall, UK, 2001.

[14] D.Q. Mayne. Control of constrained dynamic systems. European Journal of Control 7 (2001) 87-99.

[15] D.Q. Mayne, J.B. Rawlings, C.V. Rao and P.O.M. Scokaert. Constrained model predictive control: Stability and optimality. Automatica 36 (2000) 789-814.

[16] P.O.M. Scokaert and D.Q. Mayne. Min-max feedback model predictive control. IEEE Trans. Automatic Control 43:8 (1998) 1136-1142.

[17] P.O.M. Scokaert, D.Q. Mayne and J.B. Rawlings. Suboptimal model predictive control (Feasibility implies stability). IEEE Trans. Automatic Control 44:3 (1999) 648654. 